\title{
Understanding the Genetic Architecture and Gene Actions Involved in the Inheritance of Yellow Mosaic Disease and Other Yield Related Traits in Mungbean [Vigna radiata (L.) Wilczek]
}

\author{
Ch. Santhi Priya ${ }^{1}$, D. Ratna Babu
}

$10.18805 / L R-4765$

\begin{abstract}
Background: Yellow mosaic disease (YMD) transmitted by white fly, Bemisia tabaci is the most persistent and devastating biotic stress worldwide and in India resulting the reduced productivity of mungbean. At the same time the yield levels in mungbean reached a plateau, which emphasizes the importance of varieties with improved productivity.

Methods: In the present investigation, to address this urgent requirement of YMD resistant high yielding varieties, six-parameter model of generation mean analysis was employed for proper understanding of the genetic architecture and gene actions. Susceptible genotype MGG 385 was crossed with four resistant genotypes (LGG 607, LGG 630, WGG 42 and PM 5) to produce respective $F_{1} s$ (during rabi, 2018-19), $\mathrm{BC}_{1} \mathrm{~s}, \mathrm{BC}_{2} \mathrm{~s}$ (during summer, 2019) and $\mathrm{F}_{2} \mathrm{~s}$ (during kharif, 2019). These generations of the four crosses were evaluated for various yield related traits in rabi, 2019-20 and phenotyped for YMD in summer, 2020 at Lam, Guntur, Andhra Pradesh which is hotspot for YMD.

Result: The scaling tests and joint scaling test indicated the inadequacy of Additive-dominant model for all most all traits including YMD except for branches per plant and pods per cluster. The study revealed the importance of non-allelic interactions in the inheritance of majority of traits including YMD. Among the non-allelic interactions, [ $I$ ] type of interaction is predominant. Further, the control of duplicate type of epistasis in the inheritance is evident at least in few crosses for all the traits (except grain yield per plant) that had inadequacy for additive-dominant model, which hinders the pace of the progress through selection. In presence of inter-allelic interaction with such duplicate type of epistasis, population approach in self-pollinated crops, which is similar to recurrent selection in cross pollinated crops or biparental mating followed by conventional selection in the later generations should be adopted for identifying desirable segregants.
\end{abstract}

Key words: Additive-Dominant model, Duplicate epistasis, Generation means, Scaling tests, Vigna radiata.

\section{INTRODUCTION}

Mungbean [Vigna radiata (L.) Wilczek] is an easy digestible pulse crop and is considered as a major pulse crop in Asia since ancient times. It occupies an important position as it contains $51 \%$ carbohydrate, $24-26 \%$ protein, $4 \%$ minerals and $3 \%$ vitamins. Being rich in nutritional profile, it is an inseparable component in the diets of vast majority of population in the Indian sub-continent. However, several biotic and abiotic stresses are hindering the productivity. Among several constraints for mungbean production, yellow mosaic disease (YMD) is one of the most devastating diseases and an important biotic constraint in mungbean crop, causing 85 to 100 per cent yield loss which is transmitted by the white fly, Bemisia tabaci. This has become increasingly serious because of the lack of resistance in the existing cultivars. Nariani (1960) first observed the virus at IARI, New Delhi and reported an incidence of $20-30 \%$ at the institute area. There is a dire need to identify YMD resistant lines which in turn can be used in various breeding programmes. Various screening methods were devices to screen genotypes against different biotic and abiotic stresses at both gametophytic level (Babu and Ravikumar, 2010) and sporophytic level. However, field screening at sporophytic level in natural hot spots of the disease was
Department of Genetics and Plant Breeding, Advanced P.G. Center ANGRAU, Lam, Guntur-522 034, Andhra Pradesh.

${ }^{1}$ Department of Genetics and Plant Breeding, Agricultural College, Bapatla-522 101, Guntur, Andhra Pradesh, India.

Corresponding Author: D. Ratna Babu, Department of Genetics and Plant Breeding, Agricultural College, Bapatla-522 101, Guntur, Andhra Pradesh, India. Email: didlaratnababu@gmail.com

How to cite this article: Priya, C.S. and Babu, D.R. (2022). Understanding the Genetic Architecture and Gene Actions Involved in the Inheritance of Yellow Mosaic Disease and Other Yield Related Traits in Mungbean [Vigna radiata (L.) Wilczek]. Legume Research. DOI: $10.18805 / L R-4765$.

Submitted: 05-08-2021 Accepted: 11-12-2021 Online: 17-01-2022

proved to be more effective particularly for viral diseases like YMD.

In any crop for improvement programme of productivity, understanding the inheritance of any trait is of major importance. Estimation of gene action is the key in understanding the inheritance of a particular character. Among various approaches, Generation mean analysis (Hayman, 1958; Jinks and Jones, 1958; Mather and Jinks, 1971 ) is one of the best method to identify the different 
components of gene effects. It checks the adequacy of additive-dominant model using different scaling tests. Inadequacy of additive-dominant model indicate the operation of inter-allelic or non-allelic interactions. The type of non-allelic interaction and its magnitude can be estimated with precision using generation mean analysis. Hence, generation mean analysis was employed in present study to understand the gene actions involved in the inheritance of YMD and other yield related traits threadbare.

\section{MATERIALS AND METHODS}

\section{Plant material}

The susceptible genotype, MGG 385 was used as female and crossed with four resistant genotypes viz., LGG 607, LGG 630, WGG 42 and PM 5 following keel rupture method of emasculation and hand pollination (Fig 1) during rabi, 2018-19. The $F_{1} s$ of the above four crosses were used as males and back crossed to the respective parents to obtain four $\mathrm{BC}_{1} \mathrm{~s}$ (back cross on first/female parent) and four $\mathrm{BC}_{2} \mathrm{~s}$ (back cross on second/male parent) during summer, 2019. During kharif, 2019 the four $\mathrm{F}_{1} \mathrm{~s}$ were selfed to obtain the respective $F_{2} s$. Three sets of $F_{2} s$ for each $F_{1}$ were obtained.

The generation mean analysis for deciphering the genetic architecture and gene actions involved in the inheritance of yield traits and YMD were conducted in two separate experiments. During rabi, 2019-20, the six generations $\left(P_{1}, P_{2}, F_{1}, F_{2}, B C_{1}\right.$ and $\left.B C_{2}\right)$ of the four crosses were employed in generation mean analysis to understand gene actions involved in the inheritance of yield and yield related traits except YMD. While, during summer, 2020 the six generations of the four crosses were employed in generation mean analysis to understand gene actions involved in the inheritance of YMD under MYMV hotspot conditions.

\section{Field layout and screening against YMD}

Present investigation was taken up at Advanced P.G. Center, Lam, Guntur, Andhra Pradesh which is located at $16^{\circ} 36^{\prime} \mathrm{N}$ latitude, $80^{\circ} 43^{\prime} \mathrm{E}$ longitude and $31.5 \mathrm{~m}$ altitude. The first experiment of generation mean analysis for all the traits except YMD was carried out during rabi, 2019-20. Six populations of the four crosses were grown in Completely Randomized Block Design with two replications. The $\mathrm{P}_{1} \mathrm{~s}$, $\mathrm{P}_{2} \mathrm{~s}, \mathrm{BC}_{1} \mathrm{~s}, \mathrm{BC}_{2} \mathrm{~s}$ and $\mathrm{F}_{1} \mathrm{~s}$ were grown in two rows of three meter length, while the $\mathrm{F}_{2} \mathrm{~s}$ were grown in ten rows of three meter length so that 300 plants per population were available. The seed was sown with a spacing of $30 \mathrm{~cm}$ between rows and $10 \mathrm{~cm}$ with in rows. The required crop protection practices were followed as per the requirement.

The second experiment of generation mean analysis for YMD was conducted in similar lines but without spraying insecticides during the cropping period in order to maintain the natural whitefly population in the field. Disease incidence was guaranteed at the site of the experiment i.e., Advanced, P. G. Center, Lam, Guntur, because this area is hotspot for YMD. However, susceptible genotype LGG 450 was also grown after each five rows of test genotypes. Disease reaction was scored (Fig 2) on 1 to 9 scale given by Singh et al. (1992). The disease scoring was done at $45^{\text {th }}$ day after sowing. Susceptible check had a disease score of ' 9 ' by the $45^{\text {th }}$ day i.e., the day on which scoring was done on six populations of the four crosses.

\section{Statistical analysis}

The data collected on twelve traits viz., Yellow mosaic disease, days to $50 \%$ flowering, plant height, branches per plant, days to maturity, clusters per plant, pods per cluster, pods per plant, pod length, seeds per pod, test weight and grain yield per plant was subjected to generation mean analysis as per Singh and Chaudhary (2010). The presence or absence of epistasis was detected using four scaling tests (A, B, C and D) given by Mather (1949). Further, Joint Scaling test of Cavalli (1952) was also employed for testing the adequacy of Additive-Dominant model. Once the inadequacy of Additive-Dominant model was observed, the six genetic parameters viz., $[m],[d],[h],[i],[]]$ and [I] were estimated using six-parameter model of generation mean analysis (Hayman, 1958). Student t-test was used to test the significance of both scaling tests and the genetic parameters. While Chi-square test was used to test the significance of Cavalli's joint scaling test.

\section{RESULTS AND DISCUSSION}

The mean values with standard errors of YMD scores pertaining to the six generations belonging to the four crosses are presented in the Table 1 . None of the $F_{1} s$ had better disease score than the respective parents and all the four $F_{1} s$ recorded similar score as that of the resistant parent $\left(P_{2}\right)$ indicating the oligogenic nature of the trait. The oligogenic nature of YMD is in accordance with previously published results [Mahalingam et. al. (2018) and Sudha et al. (2013)]. None of the $F_{2} s$ are better than the $F_{1} s$ and had inbreeding depression, indicating the probable operation of dominance type of gene action for YMD inheritance. However, actual gene action will be revealed after performing the generation mean analysis.

It is pertinent from the means of other traits (Table 1) that the $F_{1} s$ of all the four crosses with respect to seeds per pod, plant height, clusters per plant and pod length were intermediate between the parents. Similarly; one cross (MGG $385 \times$ LGG 607) for branches per plant; one cross (MGG $385 \times$ LGG 630) for days to maturity; three crosses (MGG $385 \times$ LGG 607, MGG 385 x WGG 42 and MGG 385 x PM 5) for pods per plant; three crosses (MGG $385 \times$ LGG 607, MGG $385 \times$ LGG 630 and MGG $385 \times$ WGG 42) for test weight and; two crosses ((MGG $385 \times$ LGG 607 and MGG $385 \times$ WGG 42) for days to $50 \%$ flowering also had intermediate $F_{1} s$ values indicating the quantitative nature of inheritance of these traits in the mentioned crosses. The $F_{1}$ s of three crosses (MGG $385 \times$ LGG 630, MGG $385 \times$ WGG 42 and MGG $385 \times$ PM 5) for branches per plant; three crosses (MGG $385 \times$ LGG 607, MGG $385 \times$ WGG 42 and 
Table 1: Mean performance of six generations for YMD and yield related traits in mungbean.

\begin{tabular}{|c|c|c|c|c|c|c|c|}
\hline Cross No. & Cross & $\mathrm{P}_{1}$ & $\mathrm{P}_{2}$ & $\mathrm{~F}_{1}$ & $\mathrm{BC}_{1}$ & $\mathrm{BC}_{2}$ & $\mathrm{~F}_{2}$ \\
\hline \multicolumn{8}{|c|}{ Yellow mosaic disease } \\
\hline 1 & MGG $385 \times$ LGG 607 & $8.90 \pm 0.10$ & $1.80 \pm 0.10$ & $1.80 \pm 0.13$ & $4.10 \pm 0.13$ & $1.90 \pm 0.10$ & $3.34 \pm 0.33$ \\
\hline 2 & MGG $385 \times$ LGG 630 & $8.90 \pm 0.10$ & $2.00 \pm 0.10$ & $2.10 \pm 0.13$ & $4.40 \pm 0.10$ & $2.10 \pm 0.13$ & $3.60 \pm 0.31$ \\
\hline 3 & MGG $385 \times$ WGG 42 & $8.90 \pm 0.10$ & $3.20 \pm 0.13$ & $3.60 \pm 0.13$ & $5.90 \pm 0.10$ & $3.40 \pm 0.10$ & $5.45 \pm 0.33$ \\
\hline 4 & MGG $385 \times$ PM 5 & $8.80 \pm 0.13$ & $1.80 \pm 0.10$ & $2.00 \pm 0.13$ & $4.60 \pm 0.10$ & $2.00 \pm 0.13$ & $3.90 \pm 0.33$ \\
\hline \multicolumn{8}{|c|}{ Days to $50 \%$ flowering } \\
\hline 1 & MGG $385 \times$ LGG 607 & $32.00 \pm 0.16$ & $29.50 \pm 0.24$ & $30.00 \pm 0.16$ & $33.00 \pm 0.42$ & $33.00 \pm 0.43$ & $30.50 \pm 0.19$ \\
\hline 2 & MGG $385 \times$ LGG 630 & $32.00 \pm 0.20$ & $33.00 \pm 0.14$ & $30.50 \pm 0.22$ & $33.50 \pm 0.23$ & $33.50 \pm 0.24$ & $33.00 \pm 0.20$ \\
\hline 3 & MGG $385 \times$ WGG 42 & $35.50 \pm 0.16$ & $29.50 \pm 0.15$ & $33.50 \pm 0.19$ & $35.00 \pm 0.29$ & $34.00 \pm 0.28$ & $34.00 \pm 0.20$ \\
\hline 4 & MGG $385 \times$ PM 5 & $36.00 \pm 0.16$ & $30.50 \pm 0.20$ & $30.00 \pm 0.22$ & $34.00 \pm 0.28$ & $32.50 \pm 0.30$ & $32.50 \pm 0.22$ \\
\hline \multicolumn{8}{|c|}{ Plant height (cm) } \\
\hline 1 & MGG $385 \times$ LGG 607 & $52.30 \pm 1.04$ & $45.80 \pm 0.92$ & $48.60 \pm 0.95$ & $48.50 \pm 1.19$ & $43.20 \pm 1.12$ & $48.00 \pm 0.37$ \\
\hline 2 & MGG $385 \times$ LGG 630 & $51.60 \pm 1.01$ & $52.80 \pm 0.70$ & $52.00 \pm 1.13$ & $50.20 \pm 0.87$ & $51.00 \pm 1.14$ & $47.00 \pm 0.38$ \\
\hline 3 & MGG $385 \times$ WGG 42 & $55.10 \pm 0.87$ & $46.40 \pm 1.23$ & $50.20 \pm 1.22$ & $48.90 \pm 1.21$ & $45.00 \pm 1.24$ & $47.80 \pm 0.51$ \\
\hline 4 & MGG $385 \times$ PM 5 & $54.90 \pm 1.30$ & $47.70 \pm 1.36$ & $49.90 \pm 1.30$ & $53.61 \pm 1.15$ & $47.40 \pm 1.26$ & $47.00 \pm 0.35$ \\
\hline \multicolumn{8}{|c|}{ Branches per plant } \\
\hline 1 & MGG $385 \times$ LGG 607 & $6.40 \pm 0.35$ & $4.90 \pm 0.36$ & $5.50 \pm 0.41$ & $5.80 \pm 0.39$ & $4.80 \pm 0.32$ & $5.37 \pm 0.38$ \\
\hline 2 & MGG $385 \times$ LGG 630 & $6.10 \pm 0.40$ & $6.20 \pm 0.34$ & $6.80 \pm 0.39$ & $6.00 \pm 0.29$ & $6.60 \pm 0.22$ & $6.00 \pm 0.15$ \\
\hline 3 & MGG $385 \times$ W GG 42 & $5.90 \pm 0.39$ & $5.90 \pm 0.43$ & $6.20 \pm 0.43$ & $5.89 \pm 0.39$ & $5.90 \pm 0.29$ & $5.37 \pm 0.23$ \\
\hline 4 & MGG $385 \times$ PM 5 & $6.00 \pm 0.38$ & $6.00 \pm 0.47$ & $6.12 \pm 0.42$ & $6.00 \pm 0.26$ & $5.87 \pm 0.45$ & $5.82 \pm 0.38$ \\
\hline \multicolumn{8}{|c|}{ Days to maturity } \\
\hline 1 & MGG $385 \times$ LGG 607 & $67.50 \pm 0.23$ & $66.50 \pm 0.22$ & $65.00 \pm 0.23$ & $67.40 \pm 0.35$ & $66.00 \pm 0.45$ & $66.00 \pm 0.22$ \\
\hline 2 & MGG $385 \times$ LGG 630 & $65.00 \pm 0.20$ & $67.50 \pm 0.18$ & $66.00 \pm 0.20$ & $67.50 \pm 0.27$ & $67.00 \pm 0.26$ & $62.50 \pm 0.37$ \\
\hline 3 & MGG $385 \times$ W GG 42 & $67.50 \pm 0.24$ & $62.00 \pm 0.22$ & $60.50 \pm 0.23$ & $64.00 \pm 0.28$ & $62.50 \pm 0.28$ & $62.50 \pm 0.38$ \\
\hline 4 & MGG $385 \times$ PM 5 & $69.50 \pm 0.21$ & $61.00 \pm 0.08$ & $60.50 \pm 0.07$ & $66.00 \pm 0.29$ & $62.50 \pm 0.28$ & $62.50 \pm 0.38$ \\
\hline \multicolumn{8}{|c|}{ Clusters per plant } \\
\hline 1 & MGG $385 \times$ LGG 607 & $9.40 \pm 0.27$ & $5.10 \pm 0.24$ & $6.50 \pm 0.26$ & $7.40 \pm 0.29$ & $7.30 \pm 0.35$ & $5.37 \pm 0.38$ \\
\hline 2 & MGG $385 \times$ LGG 630 & $10.20 \pm 0.50$ & $8.10 \pm 0.36$ & $9.30 \pm 0.47$ & $9.80 \pm 0.37$ & $7.90 \pm 0.29$ & $7.08 \pm 0.22$ \\
\hline 3 & MGG $385 \times$ W GG 42 & $11.00 \pm 0.44$ & $5.30 \pm 0.45$ & $9.20 \pm 0.44$ & $9.80 \pm 0.39$ & $5.70 \pm 0.39$ & $6.74 \pm 0.19$ \\
\hline 4 & MGG $385 \times$ PM 5 & $11.00 \pm 0.14$ & $5.50 \pm 0.07$ & $10.10 \pm 0.39$ & $10.40 \pm 0.50$ & $6.90 \pm 0.47$ & $9.32 \pm 0.18$ \\
\hline \multicolumn{8}{|c|}{ Pods per cluster } \\
\hline 1 & MGG $385 \times$ LGG 607 & $3.60 \pm 0.22$ & $4.40 \pm 0.32$ & $4.62 \pm 0.22$ & $3.74 \pm 0.17$ & $4.54 \pm 0.24$ & $3.97 \pm 0.38$ \\
\hline 2 & MGG $385 \times$ LGG 630 & $3.40 \pm 0.27$ & $3.70 \pm 0.32$ & $4.40 \pm 0.32$ & $3.90 \pm 0.24$ & $4.30 \pm 0.32$ & $3.50 \pm 0.35$ \\
\hline 3 & MGG $385 \times$ W GG 42 & $3.30 \pm 0.22$ & $4.10 \pm 0.29$ & $4.30 \pm 0.30$ & $3.37 \pm 0.35$ & $4.00 \pm 0.35$ & $4.24 \pm 0.25$ \\
\hline 4 & MGG $385 \times$ PM 5 & $3.90 \pm 0.39$ & $3.00 \pm 0.35$ & $4.01 \pm 0.35$ & $3.90 \pm 0.33$ & $2.93 \pm 0.43$ & $3.52 \pm 0.32$ \\
\hline \multicolumn{8}{|c|}{ Pods per plant } \\
\hline 1 & MGG $385 \times$ LGG 607 & $32.40 \pm 0.71$ & $17.80 \pm 0.66$ & $29.00 \pm 0.78$ & $30.60 \pm 0.88$ & $16.40 \pm 0.57$ & $29.00 \pm 0.38$ \\
\hline 2 & MGG $385 \times$ LGG 630 & $34.30 \pm 0.85$ & $32.60 \pm 0.64$ & $35.70 \pm 0.87$ & $25.10 \pm 0.93$ & $31.20 \pm 0.90$ & $33.00 \pm 0.45$ \\
\hline 3 & MGG $385 \times$ WGG 42 & $34.70 \pm 0.80$ & $25.80 \pm 0.78$ & $32.60 \pm 0.94$ & $31.70 \pm 0.85$ & $25.00 \pm 0.65$ & $28.00 \pm 0.47$ \\
\hline 4 & MGG $385 \times$ PM 5 & $35.10 \pm 1.09$ & $16.00 \pm 1.20$ & $34.82 \pm 1.40$ & $31.30 \pm 1.16$ & $13.86 \pm 1.01$ & $28.30 \pm 0.58$ \\
\hline \multicolumn{8}{|c|}{ Pod length (cm) } \\
\hline 1 & MGG $385 \times$ LGG 607 & $7.22 \pm 0.13$ & $6.09 \pm 0.06$ & $7.00 \pm 0.13$ & $8.07 \pm 0.19$ & $7.94 \pm 0.17$ & $6.74 \pm 0.38$ \\
\hline 2 & MGG $385 \times$ LGG 630 & $8.32 \pm 0.12$ & $7.30 \pm 0.09$ & $7.99 \pm 0.09$ & $8.01 \pm 0.12$ & $7.95 \pm 0.10$ & $6.52 \pm 0.05$ \\
\hline 3 & MGG $385 \times$ W GG 42 & $8.38 \pm 0.10$ & $8.97 \pm 0.10$ & $8.96 \pm 0.09$ & $8.41 \pm 0.11$ & $8.82 \pm 0.11$ & $8.31 \pm 0.13$ \\
\hline 4 & MGG $385 \times$ PM 5 & $8.42 \pm 0.12$ & $9.04 \pm 0.08$ & $8.94 \pm 0.09$ & $8.00 \pm 0.12$ & $8.56 \pm 0.14$ & $8.57 \pm 0.13$ \\
\hline \multicolumn{8}{|c|}{ Seeds per pod } \\
\hline 1 & MGG $385 \times$ LGG 607 & $12.80 \pm 0.32$ & $10.80 \pm 0.45$ & $11.20 \pm 0.36$ & $12.34 \pm 0.59$ & $10.56 \pm 0.47$ & $10.06 \pm 0.38$ \\
\hline 2 & MGG $385 \times$ LGG 630 & $13.10 \pm 0.37$ & $11.10 \pm 0.46$ & $12.20 \pm 0.41$ & $11.50 \pm 0.47$ & $12.30 \pm 0.39$ & $11.00 \pm 0.15$ \\
\hline 3 & MGG $385 \times$ W GG 42 & $13.90 \pm 0.40$ & $11.90 \pm 0.52$ & $12.70 \pm 0.34$ & $11.50 \pm 0.69$ & $11.90 \pm 0.71$ & $10.30 \pm 0.31$ \\
\hline 4 & MGG $385 \times$ PM 5 & $13.80 \pm 0.46$ & $11.80 \pm 0.58$ & $13.50 \pm 0.57$ & $12.30 \pm 0.97$ & $11.63 \pm 0.75$ & $10.56 \pm 0.28$ \\
\hline
\end{tabular}

Table 1: Continue... 
Table 1: Continue...

\begin{tabular}{|c|c|c|c|c|c|c|c|}
\hline \multicolumn{8}{|c|}{ Test weight (g) } \\
\hline 1 & MGG $385 \times$ LGG 607 & $4.07 \pm 0.09$ & $3.37 \pm 0.08$ & $3.94 \pm 0.11$ & $4.00 \pm 0.10$ & $3.37 \pm 0.06$ & $3.90 \pm 0.38$ \\
\hline 2 & MGG $385 \times$ LGG 630 & $4.17 \pm 0.11$ & $3.28 \pm 0.06$ & $3.88 \pm 0.09$ & $4.02 \pm 0.11$ & $3.43 \pm 0.07$ & $3.26 \pm 0.05$ \\
\hline 3 & MGG $385 \times$ WGG 42 & $4.26 \pm 0.08$ & $5.02 \pm 0.08$ & $4.93 \pm 0.08$ & $4.00 \pm 0.12$ & $4.98 \pm 0.26$ & $4.61 \pm 0.11$ \\
\hline 4 & MGG $385 \times$ PM 5 & $4.32 \pm 0.14$ & $5.79 \pm 0.18$ & $5.94 \pm 0.14$ & $4.20 \pm 0.09$ & $5.73 \pm 0.14$ & $5.07 \pm 0.13$ \\
\hline \multicolumn{8}{|c|}{ Yield per plant (g) } \\
\hline 1 & MGG $385 \times$ LGG 607 & $6.70 \pm 0.48$ & $6.82 \pm 0.45$ & $6.62 \pm 0.46$ & $6.80 \pm 0.54$ & $6.23 \pm 0.55$ & $5.37 \pm 0.28$ \\
\hline 2 & MGG $385 \times$ LGG 630 & $6.82 \pm 0.47$ & $6.79 \pm 0.45$ & $6.93 \pm 0.56$ & $6.76 \pm 0.71$ & $6.30 \pm 0.71$ & $5.20 \pm 0.34$ \\
\hline 3 & MGG $385 \times$ WGG 42 & $6.83 \pm 0.38$ & $6.83 \pm 0.37$ & $6.93 \pm 0.43$ & $6.81 \pm 0.54$ & $6.98 \pm 0.46$ & $6.02 \pm 0.22$ \\
\hline 4 & MGG $385 \times$ PM 5 & $6.53 \pm 0.35$ & $6.83 \pm 0.45$ & $6.72 \pm 0.46$ & $6.53 \pm 0.56$ & $6.57 \pm 0.60$ & $5.37 \pm 0.29$ \\
\hline
\end{tabular}

MGG $385 \times$ PM 5) for days to maturity; all four crosses for pods per plant; one cross (MGG $385 \times$ PM 5) for test weight and; two crosses (MGG $385 \times$ LGG 630 and MGG $385 \times$ WGG 42) for yield per plant had superior $F_{1} s$ than the respective parent with less $F_{2}$ means indicating the probable operation of dominance for these traits in these crosses. However, exact gene action can be understood only after examining the results of generation mean analysis.

\section{Gene effects of yellow mosaic disease (YMD)}

There was significant deviation from 'zero' for at least one of the scaling tests and significantly deviated from Chisquare table values in joint scaling test (Table 2) in all the four crosses for YMD. This indicate the inadequacy of additive-dominant model and suggests the involvement of inter-allelic interactions viz., [ []$,[]]$ and $[I]$ in the inheritance of this trait. The component means (Table 3 ) derived from generation mean analysis revealed; positive and significant $[m]$ component, $[d]$ components and $[h]$ components for all the four crosses.

The estimates of dominant $x$ dominant [ $]$ gene effects are significant and higher in magnitude than that of both additive $\times$ additive [i] and additive [ $d$ ] estimates in all the four crosses indicating the operation of dominant $\times$ dominant [/] type of non-allelic interactions in the inheritance of this character. Though, additive and additive $\times$ additive gene (in one cross) effects are significant along with additive $x$ dominant effects, dominant $\times$ dominant gene effects overpower them because of their higher magnitude of estimates. Operation of such dominant $\times$ dominant gene action for this character was reported by Narasimhulu et al. (2018). Further these crosses also had significant [ $]$ and $[h]$ estimates with opposite signs (Table 3 ) indicating the operation of duplicate type of epistasis. Such duplicate type of epistasis for this trait was earlier observed by Narasimhulu et al. (2018). This duplicate epistasis hinders the improvement through selection and also act as limitation for exploitation of higher magnitudes of dominance and dominance $\times$ dominance gene effects. In presence of such dominant $\times$ dominant type of inter-allelic interaction with duplicate type of epistasis, population approach in selfpollinated crops proposed by Palmer (1953) which is similar to recurrent selection in cross pollinated crops or biparental mating followed by conventional selection in the later generations should be adopted for identifying desirable segregants.

\section{Gene effects of other yield related traits}

Generation mean analysis of yield and yield related traits except YMD, indicate that additive-dominant model is adequate only for two traits viz., number of branches per plant and pods per cluster. All other nine traits viz., days to $50 \%$ flowering, plant height, days to maturity, clusters per plant, pods per plant, pod length, seeds per pod, test weight and grain yield per plant had significance for one or more scaling tests and also had significant Chi-square values of joint scaling tests (Table 2). This clearly indicate the inadequacy of additive-dominant model in explaining the inheritance in these traits. Hence, the estimates of interallelic or non-allelic gene effects were obtained (Table 3 ) using six parameter model of generation mean analysis. In spite of having significant additive $[d]$ and dominance $[h]$ components, the non-allelic interaction overpowered them due to their higher estimates hence, had a great role in the inheritance of these twelve traits.

The trait wise observations of inter-allelic interactions (Table 3 ) indicate that, dominant $\times$ dominant [ $]$ type of gene effects are found to control the inheritance of days to maturity in all the four crosses. In case of plant height, two (MGG $385 \times$ LGG 607 and MGG $385 \times$ WGG 42) of the crosses, dominant $\times$ dominant [I] type of gene effects are found to be important and in the remaining two crosses (MGG $385 \times$ LGG 630 and MGG $385 \times$ WGG PM 5), the inheritance is under control of additive $\times$ additive [ $i$ ] gene effects. The inheritance of days to maturity in all the four crosses is under the control of [ $]$ type of inter-allelic interactions. Inheritance of clusters per plant is under the control of [ $[$ component in two crosses (MGG $385 \times$ LGG 607 and MGG $385 \times$ WGG $P M 5)$ and [i] component of non-allelic interactions in another two crosses (MGG $385 \times$ LGG 630 and MGG $385 \times$ WGG 42). In case of pods per plant it is evident that the inheritance is under influence of [ $I$ type of gene effects in all the four crosses.

The inheritance of pod length is determined by dominant $\times$ dominant [/] type of epistasis in three crosses (MGG $385 \times$ LGG 607, MGG $385 \times$ LGG 630 and MGG $385 \times$ PM 5) and 
Understanding the Genetic Architecture and Gene Actions Involved in the Inheritance of Yellow Mosaic Disease and Other Yield...

Table 2: Estimates of scaling tests and joint scaling tests of YMD and yield related traits in mungbean

\begin{tabular}{|c|c|c|c|c|c|c|}
\hline Cross no. & Cross & A & $\mathrm{B}$ & C & $\mathrm{D}$ & $\chi^{2}$ \\
\hline \multicolumn{7}{|c|}{ Yellow mosaic disease } \\
\hline 1 & MGG $385 \times$ LGG 607 & $-2.50 \pm 0.31^{* *}$ & $0.20 \pm 0.26$ & $-0.94 \pm 1.34$ & $0.68 \pm 0.67$ & $69.75^{\star *}$ \\
\hline 2 & MGG $385 \times$ LGG 630 & $-2.20 \pm 0.26^{* *}$ & $0.10 \pm 0.31$ & $-0.70 \pm 1.27$ & $0.70 \pm 0.64$ & $76.46^{* *}$ \\
\hline 3 & MGG $385 \times$ WGG 42 & $-0.70 \pm 0.26^{*}$ & $0.00 \pm 0.27$ & $2.50 \pm 1.37$ & $1.60 \pm 0.68^{*}$ & $12.22^{* *}$ \\
\hline 4 & MGG $385 \times$ PM 5 & $-1.60 \pm 0.27^{\star *}$ & $0.20 \pm 0.31$ & $1.00 \pm 1.37$ & $1.20 \pm 0.69^{*}$ & $39.44^{* *}$ \\
\hline \multicolumn{7}{|c|}{ Days to $\mathbf{5 0 \%}$ flowering } \\
\hline 1 & MGG $385 \times$ LGG 607 & $4.00 \pm 0.87^{* *}$ & $6.50 \pm 0.90^{* *}$ & $0.50 \pm 0.86$ & $-5.00 \pm 0.71^{* *}$ & $71.92^{* *}$ \\
\hline 2 & MGG $385 \times$ LGG 630 & $4.50 \pm 0.54^{* *}$ & $3.50 \pm 0.56^{* *}$ & $6.00 \pm 0.94^{* *}$ & $-1.00 \pm 0.52$ & $105.06^{* *}$ \\
\hline 3 & MGG $385 \times$ WGG 42 & $1.00 \pm 0.63$ & $-3.00 \pm 0.61^{* *}$ & $0.00 \pm 0.91$ & $1.00 \pm 0.56$ & $28.57^{* *}$ \\
\hline 4 & MGG $385 \times$ PM 5 & $2.00 \pm 0.63^{* *}$ & $4.50 \pm 0.66^{* *}$ & $1.50 \pm 1.00$ & $-2.50 \pm 0.60^{* *}$ & $51.65^{\star *}$ \\
\hline \multicolumn{7}{|c|}{ Plant height } \\
\hline 1 & MGG $385 \times$ LGG 607 & $-3.90 \pm 2.76$ & $-8.00 \pm 2.61^{* *}$ & $-3.30 \pm 2.79$ & $4.30 \pm 1.79^{*}$ & $10.59^{*}$ \\
\hline 2 & MGG $385 \times$ LGG 630 & $-3.20 \pm 2.30$ & $-2.80 \pm 2.63$ & $-20.40 \pm 2.98^{* *}$ & $-7.20 \pm 1.62^{* *}$ & $56.17^{* *}$ \\
\hline 3 & MGG $385 \times$ WGG 42 & $-7.50 \pm 2.85^{*}$ & $-6.60 \pm 3.03^{*}$ & $-10.70 \pm 3.52^{* *}$ & $1.70 \pm 2.02$ & $12.76^{* *}$ \\
\hline 4 & MGG $385 \times$ PM 5 & $2.42 \pm 2.95$ & $-2.80 \pm 3.14$ & $-14.40 \pm 3.51^{* *}$ & $-7.01 \pm 1.85^{\star *}$ & $29.44^{* *}$ \\
\hline \multicolumn{7}{|c|}{ Branches per plant } \\
\hline 1 & MGG $385 \times$ LGG 607 & $-0.30 \pm 0.95$ & $-0.80 \pm 0.84$ & $-0.82 \pm 1.79$ & $0.14 \pm 0.90$ & 0.95 \\
\hline 2 & MGG $385 \times$ LGG 630 & $-0.90 \pm 0.81$ & $0.20 \pm 0.68$ & $-1.90 \pm 1.12$ & $-0.60 \pm 0.48$ & 5.05 \\
\hline 3 & MGG $385 \times$ WGG 42 & $-0.32 \pm 0.97$ & $-0.30 \pm 0.84$ & $-2.73 \pm 1.38$ & $-1.05 \pm 0.67$ & 4.49 \\
\hline 4 & MGG $385 \times$ PM 5 & $-0.12 \pm 0.77$ & $-0.38 \pm 1.10$ & $-0.96 \pm 1.83$ & $-0.23 \pm 0.92$ & 0.32 \\
\hline \multicolumn{7}{|c|}{ Days to maturity } \\
\hline 1 & MGG $385 \times$ LGG 607 & $2.30 \pm 0.78^{* *}$ & $0.50 \pm 0.95$ & $0.00 \pm 1.03$ & $-1.40 \pm 0.71$ & $9.32^{*}$ \\
\hline 2 & MGG $385 \times$ LGG 630 & $4.00 \pm 0.61^{* *}$ & $0.50 \pm 0.59$ & $-14.50 \pm 1.54^{* *}$ & $-9.50 \pm 0.82^{* *}$ & $152.09^{* *}$ \\
\hline 3 & MGG $385 \times$ WGG 42 & $0.00 \pm 0.66$ & $2.50 \pm 0.65^{\star *}$ & $-0.50 \pm 1.61$ & $-1.50 \pm 0.85$ & $15.92^{* *}$ \\
\hline 4 & MGG $385 \times$ PM 5 & $2.00 \pm 0.62^{* *}$ & $3.50 \pm 0.57^{* *}$ & $-1.50 \pm 1.55$ & $-3.50 \pm 0.86^{* *}$ & $49.36^{* *}$ \\
\hline \multicolumn{7}{|c|}{ Clusters per plant } \\
\hline 1 & MGG $385 \times$ LGG 607 & $-1.10 \pm 0.70$ & $3.00 \pm 0.78^{* *}$ & $-6.03 \pm 1.64^{* *}$ & $-3.96 \pm 0.88^{* *}$ & $35.35^{\star *}$ \\
\hline 2 & MGG $385 \times$ LGG 630 & $0.10 \pm 1.02$ & $-1.60 \pm 0.83$ & $-8.58 \pm 1.43^{* *}$ & $-3.54 \pm 0.65^{\star *}$ & $48.37^{* *}$ \\
\hline 3 & MGG $385 \times$ WGG 42 & $-0.60 \pm 0.99$ & $-3.10 \pm 0.99^{* *}$ & $-7.74 \pm 1.31^{* *}$ & $-2.02 \pm 0.67^{\star *}$ & $39.97^{* *}$ \\
\hline 4 & MGG $385 \times$ PM 5 & $-0.30 \pm 1.08$ & $4.20 \pm 1.03^{* *}$ & $0.58 \pm 1.06$ & $-1.66 \pm 0.77^{\star}$ & $17.60^{* *}$ \\
\hline \multicolumn{7}{|c|}{ Pods per cluster } \\
\hline 1 & MGG $385 \times$ LGG 607 & $-0.74 \pm 0.47$ & $0.06 \pm 0.62$ & $-1.36 \pm 1.62$ & $-0.34 \pm 0.81$ & 3.00 \\
\hline 2 & MGG $385 \times$ LGG 630 & $0.00 \pm 0.64$ & $0.50 \pm 0.77$ & $-1.90 \pm 1.59$ & $-1.20 \pm 0.80$ & 2.41 \\
\hline 3 & MGG $385 \times$ WGG 42 & $-0.86 \pm 0.79$ & $-0.40 \pm 0.81$ & $0.96 \pm 1.21$ & $1.11 \pm 0.69$ & 2.78 \\
\hline 4 & MGG $385 \times$ PM 5 & $-0.11 \pm 0.85$ & $-1.15 \pm 0.99$ & $-0.84 \pm 1.54$ & $0.21 \pm 0.83$ & 1.43 \\
\hline \multicolumn{7}{|c|}{ Pods per plant } \\
\hline 1 & MGG $385 \times$ LGG 607 & $-0.20 \pm 2.05$ & $-14.00 \pm 1.53^{* *}$ & $7.80 \pm 2.38^{* *}$ & $11.00 \pm 1.29^{\star *}$ & $153.84^{* *}$ \\
\hline 2 & MGG $385 \times$ LGG 630 & $-19.80 \pm 2.23^{* *}$ & $-5.90 \pm 2.11^{\star *}$ & $-6.30 \pm 2.73^{*}$ & $9.70 \pm 1.58^{* *}$ & $82.95^{\star *}$ \\
\hline 3 & MGG $385 \times$ WGG 42 & $-3.90 \pm 2.10$ & $-8.40 \pm 1.79^{* *}$ & $-13.70 \pm 2.88^{* *}$ & $-0.70 \pm 1.42$ & $30.62^{* *}$ \\
\hline 4 & MGG $385 \times$ PM 5 & $-7.32 \pm 2.92^{*}$ & $-23.10 \pm 2.73^{* *}$ & $-7.54 \pm 3.99$ & $11.44 \pm 1.93^{* *}$ & $80.28^{* *}$ \\
\hline \multicolumn{7}{|l|}{ Pod length } \\
\hline 1 & MGG $385 \times$ LGG 607 & $4.00 \pm 0.42^{* *}$ & $2.79 \pm 0.37^{* *}$ & $-0.35 \pm 1.54$ & $-2.53 \pm 0.80^{\star *}$ & $72.10^{* *}$ \\
\hline 2 & MGG $385 \times$ LGG 630 & $4.50 \pm 0.28$ & $0.61 \pm 0.24^{*}$ & $-5.52 \pm 0.30^{* *}$ & $-2.92 \pm 0.18^{\star *}$ & $459.10^{* *}$ \\
\hline 3 & MGG $385 \times$ WGG 42 & $1.00 \pm 0.27$ & $-0.31 \pm 0.26$ & $-2.07 \pm 0.57^{\star *}$ & $-0.61 \pm 0.31^{*}$ & $15.41^{* *}$ \\
\hline 4 & MGG $385 \times$ PM 5 & $2.00 \pm 0.28^{* *}$ & $-0.86 \pm 0.30^{* *}$ & $-1.06 \pm 0.58$ & $0.58 \pm 0.32$ & $30.77^{* *}$ \\
\hline \multicolumn{7}{|c|}{ Seeds per pod } \\
\hline 1 & MGG $385 \times$ LGG 607 & $0.68 \pm 1.28$ & $-0.88 \pm 1.10$ & $-5.76 \pm 1.76^{* *}$ & $-2.78 \pm 1.07^{* *}$ & $11.88^{* *}$ \\
\hline 2 & MGG $385 \times$ LGG 630 & $-2.30 \pm 1.09^{*}$ & $1.30 \pm 0.99$ & $-4.60 \pm 1.18^{* *}$ & $-1.80 \pm 0.68^{* *}$ & $28.28^{* *}$ \\
\hline 3 & MGG $385 \times$ WGG 42 & $-3.60 \pm 1.48^{*}$ & $-0.80 \pm 1.55$ & $-10.00 \pm 1.55^{\star *}$ & $-2.80 \pm 1.16^{*}$ & $44.36^{* *}$ \\
\hline 4 & MGG $385 \times$ PM 5 & $-2.70 \pm 2.08$ & $-2.04 \pm 1.72$ & $-10.36 \pm 1.77^{* *}$ & $-2.81 \pm 1.36^{*}$ & $34.85^{\star *}$ \\
\hline
\end{tabular}

Table 2: Continue... 
Table 2: Continue...

\begin{tabular}{|c|c|c|c|c|c|c|}
\hline \multicolumn{7}{|c|}{ Test weight } \\
\hline 1 & MGG $385 \times$ LGG 607 & $-0.01 \pm 0.24$ & $-0.55 \pm 0.18^{* *}$ & $0.28 \pm 1.53$ & $0.42 \pm 0.76$ & $10.58^{*}$ \\
\hline 2 & MGG $385 \times$ LGG 630 & $-0.01 \pm 0.26$ & $-0.30 \pm 0.18$ & $-2.17 \pm 0.30^{* *}$ & $-0.93 \pm 0.17^{\star *}$ & $60.17^{* *}$ \\
\hline 3 & MGG $385 \times$ WGG 42 & $-1.19 \pm 0.27^{* *}$ & $0.01 \pm 0.53$ & $-0.70 \pm 0.49$ & $0.24 \pm 0.36$ & $19.57^{* *}$ \\
\hline 4 & MGG $385 \times$ PM 5 & $-1.86 \pm 0.27^{\star *}$ & $-0.27 \pm 0.37$ & $-1.71 \pm 0.63^{* *}$ & $0.21 \pm 0.31$ & $47.68^{* *}$ \\
\hline \multicolumn{7}{|c|}{ Grain yield per plant } \\
\hline 1 & MGG $385 \times$ LGG 607 & $0.28 \pm 1.27$ & $-0.98 \pm 1.27$ & $-5.29 \pm 1.60^{* *}$ & $-2.29 \pm 0.95^{\star}$ & $12.88^{* *}$ \\
\hline 2 & MGG $385 \times$ LGG 630 & $-0.23 \pm 1.60$ & $-1.12 \pm 1.59$ & $-6.67 \pm 1.89^{* *}$ & $-2.66 \pm 1.22^{*}$ & $13.22^{* *}$ \\
\hline 3 & MGG $385 \times$ WGG 42 & $-0.14 \pm 1.22$ & $0.20 \pm 1.07$ & $-3.44 \pm 1.33^{*}$ & $-1.75 \pm 0.83^{*}$ & $8.53^{*}$ \\
\hline 4 & MGG $385 \times$ PM 5 & $-0.17 \pm 1.25$ & $-0.41 \pm 1.36$ & $-5.33 \pm 1.60^{* *}$ & $-2.37 \pm 1.00^{*}$ & $12.19^{* *}$ \\
\hline 5 & LGG $607 \times$ LGG 630 & $0.14 \pm 1.25$ & $0.08 \pm 1.25$ & $-3.56 \pm 1.39^{*}$ & $-1.89 \pm 0.89^{*}$ & $8.52^{*}$ \\
\hline
\end{tabular}

${ }^{*}$ and ${ }^{* *}$ represents $5 \%$ and $1 \%$ level of significance, respectively.
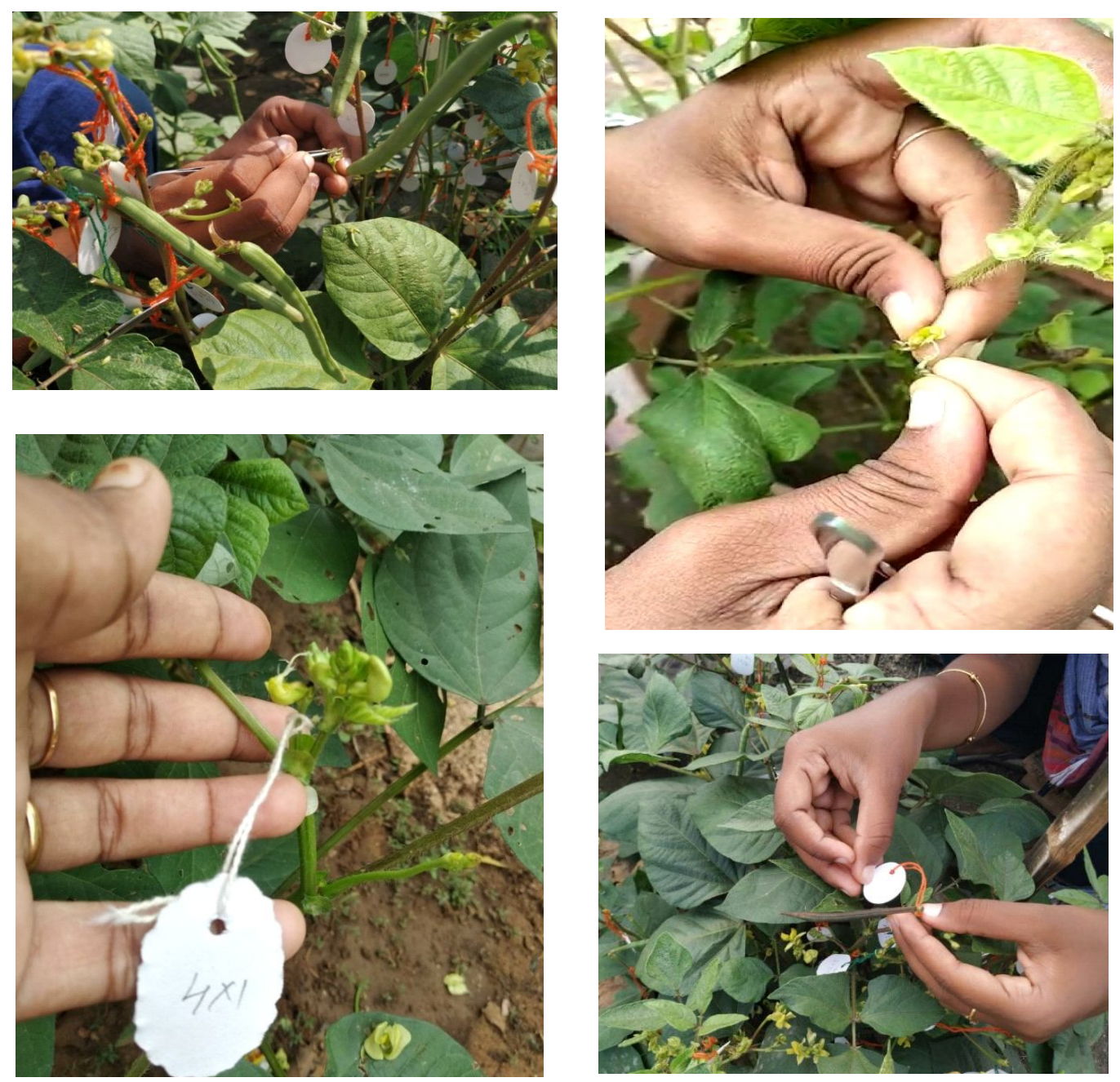

Fig 1: Crossing technique a. Emasculation, b. Pollination, c. Tagging and d. Developed pod from crossed flower.

by additive $\times$ additive [i] type of epistasis in the remaining one crosses (MGG $385 \times$ WGG 42). The trait, seeds per pod is under control of additive $\times$ additive [i] type of gene effects in all the four crosses. Test weight's inheritance is under influence of; dominant $x$ dominant [ $I$ gene effects in one cross (MGG $385 \times$ PM 5); additive $\times$ additive [i] gene effects in one cross (MGG $385 \times$ LGG 630) and; additive $\times$ dominant [] gene effects in the remaining two crosses (MGG $385 \times$ LGG 607 and MGG $385 \times$ WGG 42). With respect to grain yield per plant, all the four crosses, inheritance is under the control of additive $\times$ additive [i] type of non-allelic interactions. In spite of having significant magnitudes of other 


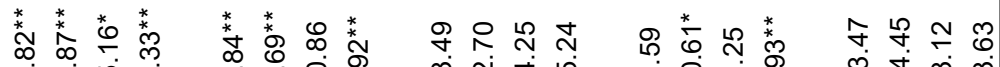

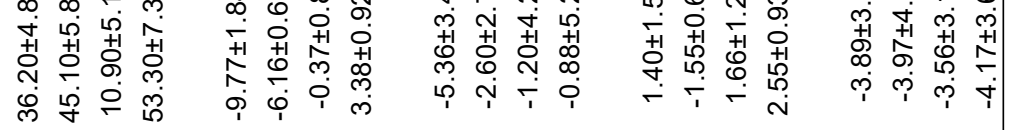

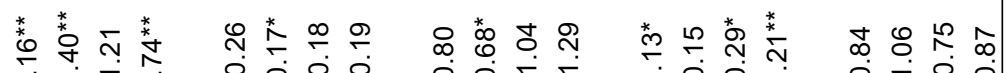

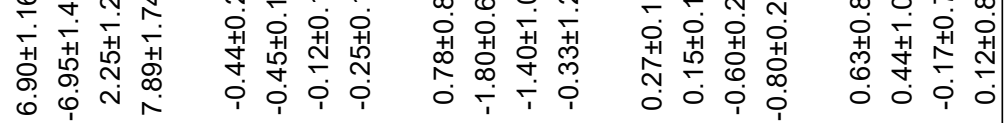

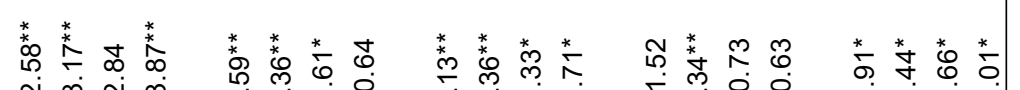

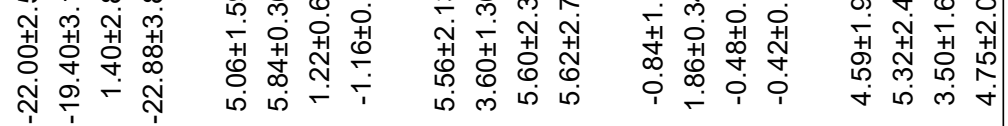

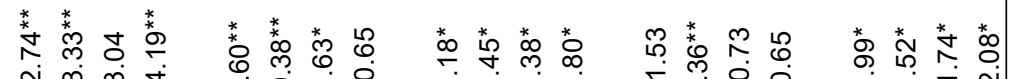

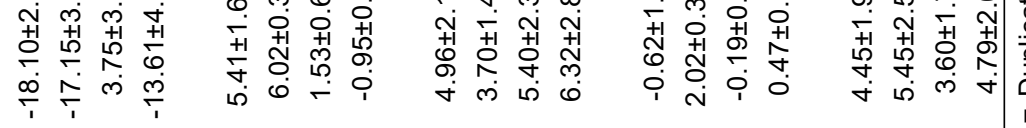

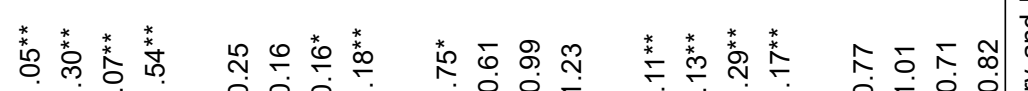

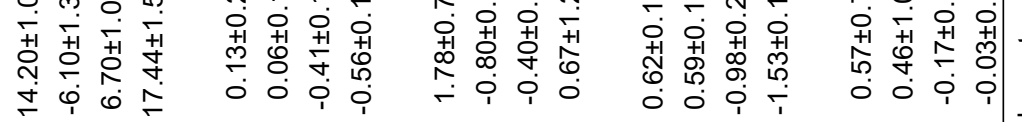

覓

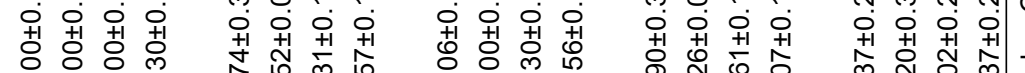
市

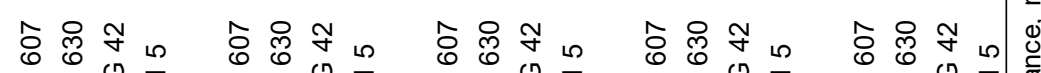

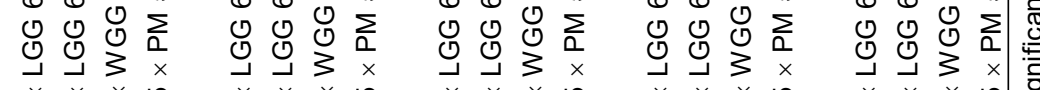

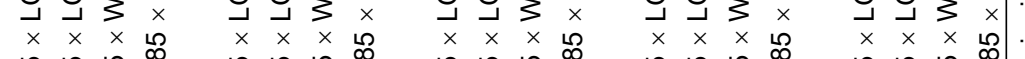

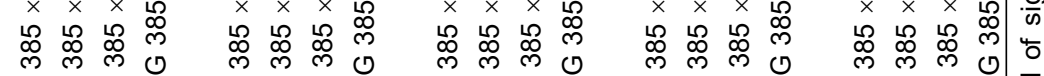

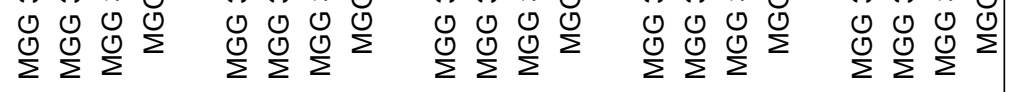

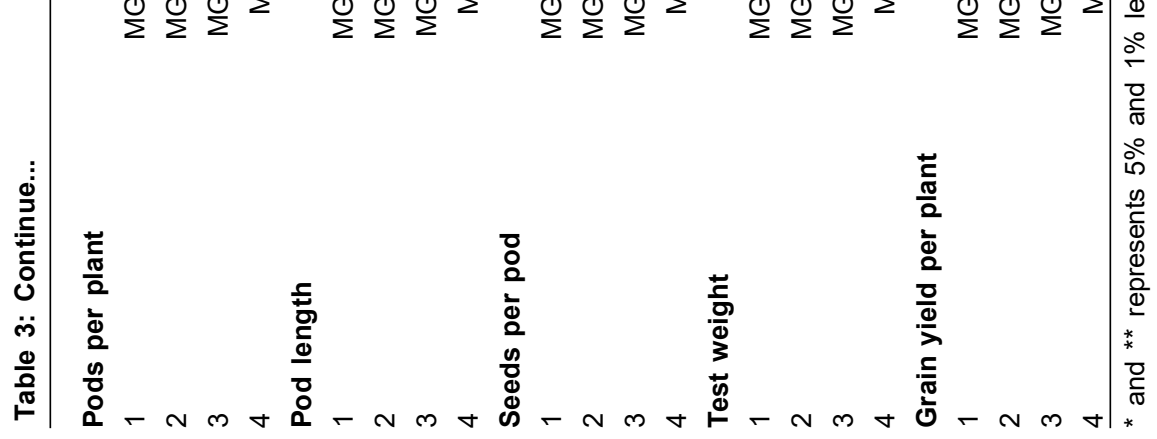




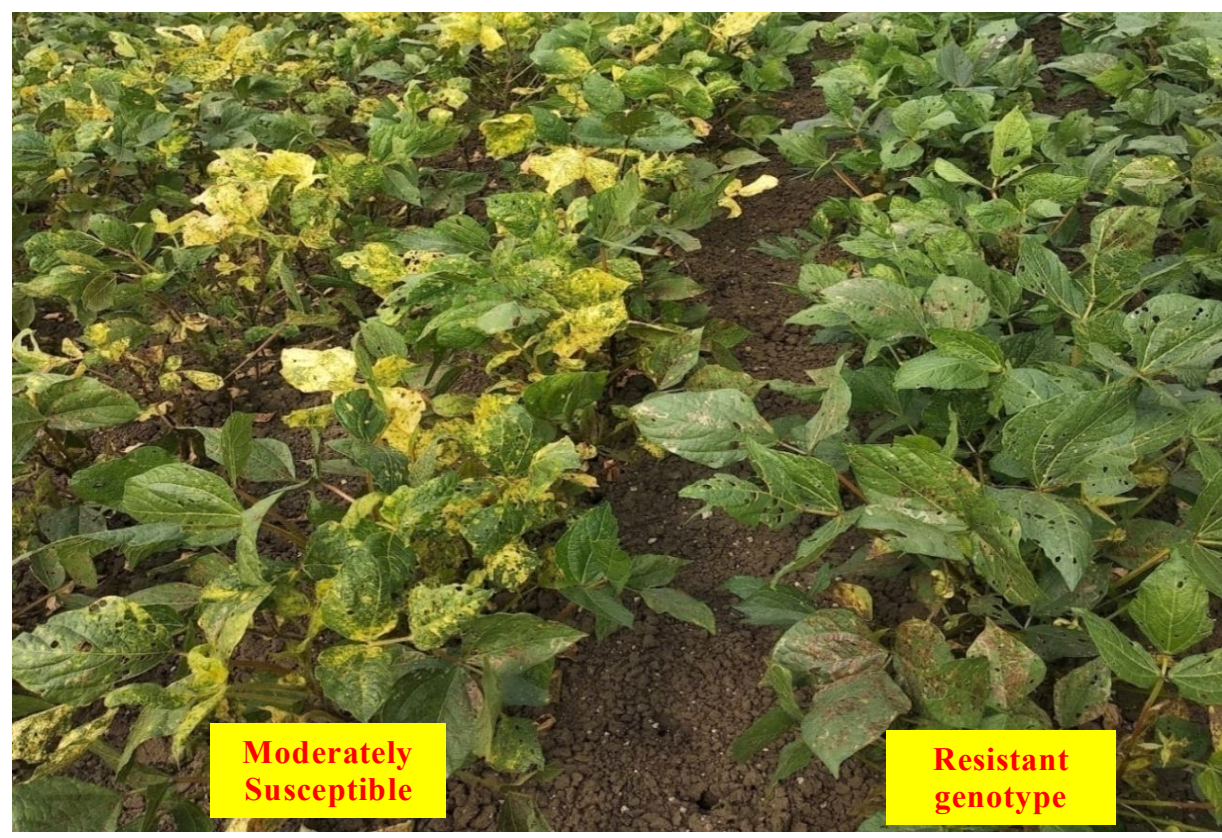

Fig 2: Screening of greengram genotypes against yellow mosaic disease.

gene effects for various traits of different crosses, the abovementioned gene effects overpowered them due to their higher magnitudes of effects.

Similar results of involvement of non-allelic or interallelic interactions in the inheritance of various traits were reported by many scientists [Khattak et al. (2004), Singh et al. (2006), Alam et al. (2014), Pathak et al. (2015), Singh et al. (2016), Narasimhulu et al. (2018), Yadav et al. (2017) and Sinha et al. (2020)] in mungbean. The observed differences in the crosses in terms of gene action for the same trait could be attributed to change in gene frequencies and proportion of dominant and recessive genes possessed by the parents involved in the crosses (Viana et al., 1999). Inadequacy of additive-dominant model for explaining the inheritance of the ten out of twelve traits emphasizes the complex nature of gene effects suggesting that simple selection procedures may not be sufficient to improve the yield and its contributing traits. Further, significant magnitudes with opposite signs of $[h]$ and [I] gene effects indicate the operation of duplicate type of epistasis. This control of duplicate type of epistasis in the inheritance is evident at least in few crosses for all the traits (except grain yield per plant) that had inadequacy for additive-dominant model. This duplicate type of epistasis was earlier indicated for various traits by different scientists [Khattak et al. (2004), Singh et al. (2006), Pathak et al. (2015), Singh et al. (2016), Narasimhulu et al. (2018), Yadav et al. (2017) and Sinha et al. (2020)]. This duplicate type of epistasis will reduce the variation in $F_{2}$ and subsequent generations, consequently hinders the pace of the progress through selection. Therefore, the best strategy to counter this duplicate epistasis in presence of non-allelic interactions is to go for inter-matings in early segregating generations and postpone the selections to the later generations.

\section{CONCLUSION}

The scaling tests and Joint Scaling test indicated the inadequacy of Additive-dominant model for all most all traits including YMD except for branches per plant and pods per cluster. The study revealed the importance of non-allelic interactions in the inheritance of majority of traits including YMD. Among the non-allelic interactions, [l] type of interaction is predominant. Further, the control of duplicate type of epistasis in the inheritance is evident at least in few crosses for all the traits (except grain yield per plant) that had inadequacy for additive-dominant model, which hinders the pace of the progress through selection. In presence of inter-allelic interaction with such duplicate type of epistasis, population approach in self-pollinated crops, which is similar to recurrent selection in cross pollinated crops or biparental mating followed by conventional selection in the later generations should be adopted for identifying desirable segregants. Finally, from the results of six parameter model of generation mean analysis it can be concluded that gene interactions varied cross wise as well as trait wise. Hence, specific breeding strategy has to be adopted in particular cross for a particular trait depending up on the type of gene effects operating, for overall improvement of yield and its contributing traits.

\section{REFERENCES}

Alam, A.M., Somta, P. and Srinives, P. (2014). Generation mean and path analysis of reaction to mungbean yellow mosaic virus (MYMV) and yield related traits in mungbean [Vigna radiata (L.) Wilczek]. SABRAO Journal of Breeding and Genetics. 46(1): 150-159.

Babu, D.R. and Ravikumar, R.L. (2010). Parallel response between gametophyte and sporophyte for Fusarium wilt resistance in the recombinant inbred lines of chickpea (Cicer arietinum L.) Current Science. 29(4): 513-518. 
Cavalli, L.L. (1952). An Analysis of Linkage in Quantitative Inheritance. In: Quantitative Inheritance. (ed. E.C.R. Reeve and C.H. Waddington). HMSO, London: 135-144.

Hayman, B.I. (1958). The separation of epistasis from additive and dominance variation in generation means. Heredity. 12 : 371-390.

Jinks, J.L and Jones, R.M. (1958). Estimation of components of heterosis. Genetics. 43: 223-234.

Khattak, G.S.S., Ashrafi, M and khan, M.S. (2004). Assessment of genetic variation for yield and yield components in mungbean [Vigna radiata (L.) Wilczek] using generation means analysis. Pakistan Journal of Botany. 36(3): 583-588.

Mahalingam, A., Satya, V.K., Manivannan, N.S., Narayanan, L. and Sathya, P. (2018). Inheritance of mungbean yellow mosaic virus disease resistance in greengram [Vigna radiata (L.) Wilczek]. International Journal of Current Microbiology and Applied Sciences. 7(1): 880-885.

Mather, K and Jinks, J. (1971). Biometrical Genetics. $3^{\text {rd }}$ edition, Chapman and Hall, London.

Mather, K. (1949). Biometrical Genetics. Dover publication Inc., New York.

Narasimhulu, R., Naidu, N.V and Reddy, K.H.P. (2018). Genetic analysis for yield and yield attributes in greengram [Vigna radiata (L.) Wilczek]. Legume Research. 41(3): 349-355.

Nariani, T.K. (1960). Yellow mosaic of mungbean (Phaseolus aureus L.). Indian Phytopathology. 13: 24-29.

Palmer, T.P. (1953). Progressive improvement in self fertilized crops. Heredity. 7: 127-129.

Pathak, N., Singh, M.N., Mishra, M.K and Saroj, S.K. (2015). Nature of gene action for yield and its components in mungbean (Vigna radiata). The Indian Journal of Agricultural Sciences. 85(4): 504-508.
Singh, R.K and Chaudhary, B.D. (2010). Biometrical Methods in Quantitative Genetic Analysis. Kalyani Publishers, New Delhi. 229-252.

Singh, I., Gill, M.S. and Bains, T.S. (2006). Generation mean analysis for yield attributing traits in mungbean [Vigna radiata (L.) Wilczek]. Indian Journal of Genetics and Plant Breeding. 66(1): 47-48.

Singh, G., Sharma, Y.R and Kaur, L.R. (1992). Method of rating mungbean yellow mosaic virus in mungbean and urdbean. Plant Disease Research. 7: 1-6.

Singh, C.M., Singh, A.K., Mishra, S.B. and Pandey, A. (2016). Generation mean analysis to estimate the genetic parameters for yield improvement and inheritance of seed colour and lusture in mungbean [Vigna radiata (L.) Wilczek]. Legume Research. 39(4): 494-501.

Sinha, S., Mishra, S.B., Kumar, A. and Pandey, S.S. (2020). Estimation of gene effects for yield attributing traits in mungbean (Vigna radiata) and urdbean (Vigna mungo) for intra and interspecific crosses. Electronic Journal of Plant Breeding. 11(1): 81-85.

Sudha, M., Karthikeyan, A., Anusuya, P., Ganesh, N.M., Pandiyan, M., Senthil, N., Raveendran, M., Nagarajan, P. and Angappan, K. (2013). Inheritance of resistance to mungbean yellow mosaic virus (MYMV) in inter and intra specific crosses of mungbean (Vigna radiata). American Journal of Plant Sciences. 4: 1924-1927.

Viana, J.M.S., Cruz, C.D and Cardoso, A.A. (1999). Theory and analysis of partial diallel crosses. Genetics and Molecular Biology. 22: 591-599.

Yadav, S.M., Prakash, V and Khedar, O.P. (2017). Gene action of yield and its contributing characters in mungbean [Vigna radiata (L.) Wilczek] under different environments. Journal of Pharmacognosy and Phytochemistry. 6(5): 999-1003. 